\title{
Evolution of the Habitable Zone and Search for Life Around Giant Stars Part II: Feasibility with Darwin/TPF
}

\author{
W. C. Danchi ${ }^{1}$, B. Lopez ${ }^{2}$, J. Schneider ${ }^{3}$, A. Belu ${ }^{2,4}$, R. Barry ${ }^{1,5}$, \\ J. Rajagopal ${ }^{1,6}$, and L. J. Richardson ${ }^{1}$ \\ ${ }^{1}$ NASA Goddard Space Flight Center, Exoplanets and Stellar Astrophysics Lab., Code 667, \\ Greenbelt, MD 20771, USA \\ ${ }^{2}$ Observatoire de la Cote dAzur, BP 4229, 06304 Nice Cedex 4, France \\ ${ }^{3}$ Observatoire de Paris, Paris, France \\ ${ }^{4}$ University of Nice - Sophia Antipolis, LUAN, Parc Valrose, 06108 Nice Cedex 2, France \\ ${ }^{5}$ The Johns Hopkins University, Physics and Astronomy Dept., Baltimore, MD 21218, USA \\ ${ }^{6}$ University of Maryland, Astronomy Department, College Park, MD 20742, USA
}

\begin{abstract}
We discuss the evolution of the habitable zone around low mass and intermediate mass stars as they evolve off the main sequence. This work shows that this new class of stars should be included in the search for life because if planets could be found in their habitable zones, and these planets showed evidence for life, it is possible to empirically determine a lower limit to the timescale for the formation of life. This time scale is not well determined from the study of the Earth (or planets around main sequence stars), as life formation initially occurred during a period of heavy bombardment from comets and asteroids during the formation of the solar system. Our initial research was recently published (Lopez, Schneider, \& Danchi 2005). We will describe our work in progress, in which we perform calculations and simulations aiming to demonstrate the potential of TPF and Darwin for the search and characterization of planets around evolved stars.
\end{abstract}

Keywords. instrumentation: high angular resolution, techniques: interferometric, space vehicles: instruments, planets and satellites: general, planetary systems: formation.

\section{Introduction and Basic Concepts}

Low and intermediate mass stars with masses ranging from 0.5 to $\sim 8 \mathrm{M}_{\odot}$ undergo dramatic changes in temperature and luminosity during their evolution. For example, the luminosity of the Sun, which is located on the Main Sequence of the Hertzprung-Russell diagram, currently is 30 percent greater than its zero-age (initial) luminosity (Gough 1981). Other major changes take place after the Sun evolves off the Main Sequence. Along the Red Giant Branch (RGB) the radius of a $1 \mathrm{M}_{\odot}$ star progressively increases up to $10 \mathrm{R}_{\odot}$, and the luminosity increases up to about $10^{1.5} \mathrm{~L}_{\odot}$ (Maeder \& Meynet 1988). Then, after leaving the RGB a star evolves onto the Asymptotic Giant Branch (AGB). At this stage in its evolution, its radius increases up to a few hundred times RSun and the luminosity up to nearly $10^{4} \mathrm{~L}_{\odot}$. The lifetime of a $1 \mathrm{M}_{\odot}$ star, from the moment it leaves the main sequence until the end of the RGB, is about $2.5 \times 10^{9} \mathrm{yr}$, which can be compared to $\sim 10 \times 10^{9}$ yr spent on the main sequence. The evolution of a star is accompanied by mass loss processes that play an important role. During the post-main sequence evolution of a $1 \mathrm{M}_{\odot}$ star nearly $0.4 \mathrm{M}_{\odot}$ will be lost to the interstellar medium before it ends its life as a white dwarf (Weidemann 1987). 
Table 1. Lifetimes (Gyr) of stages of stellar evolution as a function of initial stellar mass

\begin{tabular}{ccccc}
\hline Mass $\left(\mathrm{M}_{\odot}\right)$ & Main Sequence & Subgiant & First Red Giant & Core He Burning \\
\hline 1.0 & 7.41 & 2.63 & 1.45 & 0.95 \\
1.5 & 1.72 & 0.41 & 0.18 & 0.26 \\
2.0 & 0.67 & 0.11 & 0.04 & 0.10 \\
\hline
\end{tabular}

The habitable zone is conservatively defined as a shell region around a star where liquid water can be found at the surface of a planet. This zone is typically from about $0.95 \mathrm{AU}$ to $1.37 \mathrm{AU}$ for G stars (Kasting et al. 1993, Forget 1998). A less conservatively defined habitable zone extends the outer edge of the limit of the habitable zone to as large as $2.4 \mathrm{AU}$, depending on the radiative properties of $\mathrm{CO}_{2}$ ice clouds (Forget \& Pierrehumbert 1997, Mischna et al. 2000). Consequently, as the temperature and luminosity of a star changes during the course its evolution, the radius and width of this zone will also evolve with time.

The habitable zone gradually moves outward after a star leaves the main sequence. Depending on the length of time that is necessary for life to form, we show that it is possible that life may exist or develop on a planet that is located within a range of 2-9 AU from a $1 \mathrm{M}_{\odot}$ star, during the first stages of its post main-sequence evolution and up to $22 \mathrm{AU}$ from the star during the second stage after the core He flash. It is possible that the effects of natural migration of micro-organisms through meteorites may be a possible trigger for the emergence of life around evolved stars (Arrhenius 1903, O'Keefe et al. 1996, Zubrin 2001). Micro-organisms might also transport life from one planet to another planet during the evolution of a star. For example, they could be transported from a planet where life is ending to a more distant planet where favorable conditions for the re-birth of life are encountered because of the sufficiently slow evolution of the habitable zone.

Future space missions, such as TPF (Beichman, Woolf, \& Lindensmith 1999) and Darwin (Fridlund \& Gondoin 2003), that focus on searches for signatures of life on extrasolar planets primarily around main-sequence stars, should also consider the environments of sub-giant and red giant stars. Pathfinder missions such as EPIC (Clampin et al. 2001), Eclipse (Trauger et al. 2003), and FKSI (Danchi et al. 2003a, 2003b) may also be able to observe planets around red giant stars.

In this short paper we discuss two main issues related to the evolution of the habitable zone. The first is some of the characteristics of the subgiant and giant stars in the solar neighborhood. The second is the feasibility of detection of earth-like planets around these stars by comparing these stars to the current star list for the interferometer version of TPF or Darwin.

\section{Characteristics of subgiant and red giant stars in the Solar neighborhood}

Figure 1 displays an empirical Hertzprung-Russell diagram for stars in the globular cluster M3 that we have modified from Shu (1982). The focus of our work on the evolution of the habitable zone is on stars that have evolved off the main sequence into the subgiant or red giant branches, and NOT the extremely large and luminous Asymptotic Giant Branch (AGB) stars. Common misconceptions are based on the fact that many astronomers automatically think only of AGB stars in the context of evolved stars. Thus they think that red giant stars are too cool, too luminous, and too large to be worth 


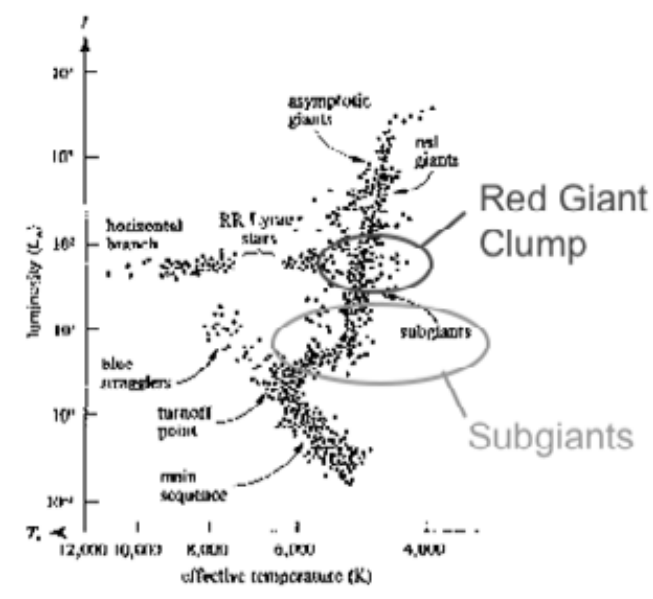

\section{Empirical HR Diagram}

Figure 1. Hertzprung-Russell diagram of the globular cluster M3. Adapted from Shu (1982).

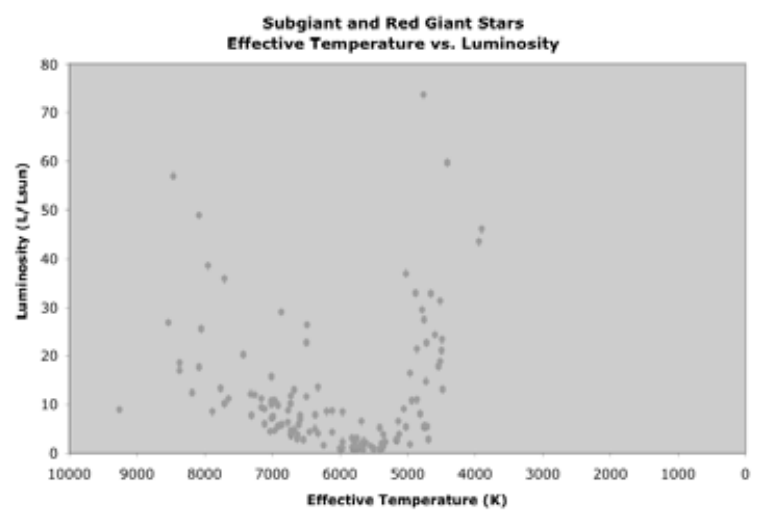

Figure 2. Temperature versus luminosity for the approximately 130 nearby luminosity class III and IV stars within 30 pc of our solar system.

studying in the context of life formation and evolution. As we shall see in the remainder of this section, these misconceptions are false, and a reasonable sample of such stars exists and are observable with TPF or Darwin without modification from what is planned for the main sequence stars.

Figures 2-4 display some of the characteristic features of luminosity class IV and III stars within $30 \mathrm{pc}$ of the Solar system. The sample of stars in these figures was extracted from the catalog of 2350 stars within 30 pc that was created for the Terrestrial Planet Finder mission. This list contains approximately 120 stars and is essentially the same set of stars that were published in Lopez, Schneider, and Danchi (2005) in their Tables II-IV. The master list for TPF, however, compiles much useful physical information, which we have used in this paper to compare with main sequence luminosity class V stars that are the main focus of the TPF mission.

It is easy to see from Figure 2 that the subgiant and red giant stars of interest to this work have only modestly higher luminosities and roughly the same effective temperatures of the main sequence stars. Figure 3 displays a histogram of the luminosities of the 


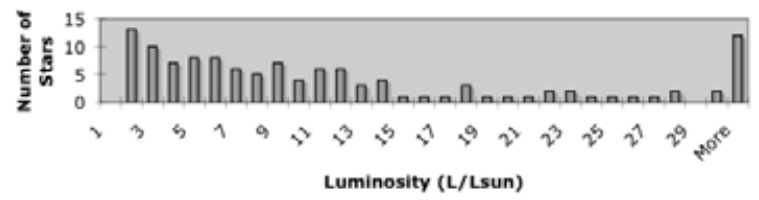

Figure 3. Luminosity distribution for the luminosity class III and IV stars in the solar neighborhood. Note the distribution is peaked at luminosities in the range of $3-5 \mathrm{~L}_{\odot}$.

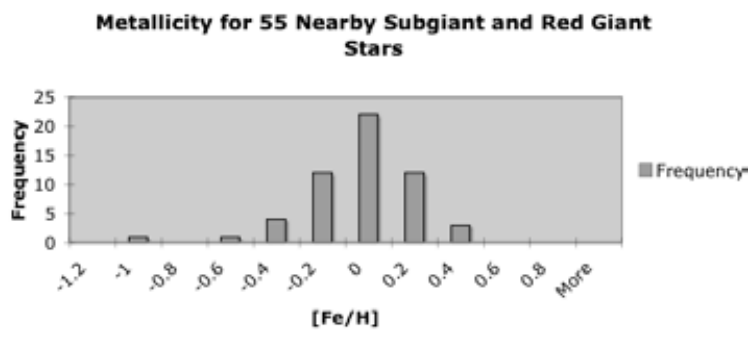

Figure 4. Distribution of metallicities for 55 luminosity class IV and III stars within $30 \mathrm{pc}$ of the solar system extracted from the approximately 130 stars shown in the preceding figures.

selected stars. It is clear that the bulk of the stars have luminosities in the range of 3-10 $\mathrm{L}_{\odot}$, and hence their stellar radii are in the range of $\sim 1.7-3.3 \mathrm{R}_{\odot}$.

Figure 4 displays metallicities compared to solar values for 55 stars of the larger sample. These stars were the only ones with metallicities that were available in TPF star list database. These stars show a peak in the metallicity distribution about 0.1 dex larger than solar metallicity.

\section{Feasibility of observations with TPF and Darwin}

The signal-to-noise ratio for detecting planets around stars using a nulling interferometer depends on a number of factors. The signal term is simply the photon rate for the planet $R_{\text {planet }}$ times the integration time, T. Similarly, the noise terms include the stellar leakage signal $\left(R_{S L}\right)$, the presence of exo-zodial dust $\left(R_{E Z}\right)$, our own local zodiacal foreground $\left(R_{L Z}\right)$, and thermal noise $\left(R_{t h}\right)$ from the telescope and instrument itself, where these quantities are also multiplied by the integration time to obtain the total number of noise photons. The signal-to-noise is thus:

$$
S N R=\frac{\text { Planet Signal }}{\text { Noise }} \propto \frac{R_{\text {planet }} T}{\left[\left(R_{S L}+R_{L Z}+R_{E Z}+R_{t h}\right) T\right]^{1 / 2}},
$$

where we follow the notation of Dubovitsky and Lay (2004). The present architecture studies for the interferometer version of TPF and Darwin indicate that under most circumstances the noise terms for the extra-solar zodiacal noise can be neglected if the total flux is less than about 5 times the contribution from the planet signal (Dubovitsky \& Lay 2004). The term for the thermal noise is usually small compared to that from the local zodiacal emission; this is normally done as a requirement on the temperature of the telescope itself, and is why TPF and Darwin need to be cooled to about 35-40 K. These considerations imply that the dominant terms for the noise are the local zodiacal foreground and the stellar leakage. Clearly for the subgiant and red giant stars, the local 


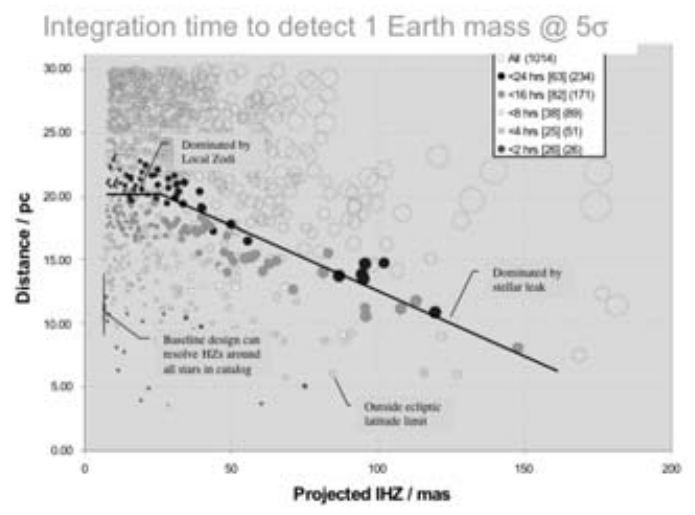

Figure 5. Integration time estimates for the Terrestrial Planet Finder Interferometer (TPF-I) as a function of projected inner habitable zone in milli-arcseconds (mas) and distance in parsecs (pc) from our solar system (Dubovitsky \& Lay 2004).

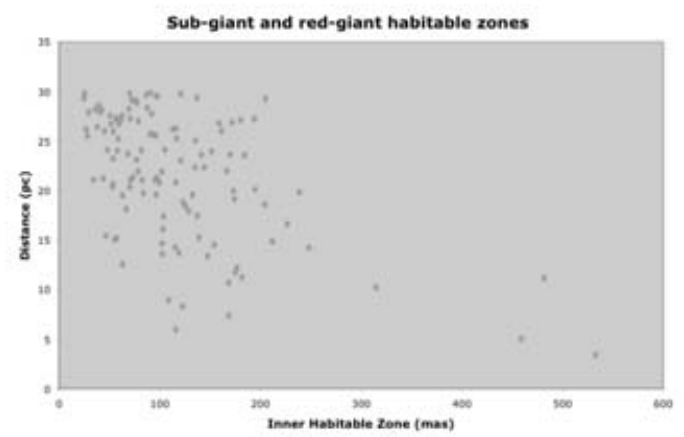

Figure 6. Inner habitable zone radius (mas) versus distance (pc) for the stars in Fig. 3.

zodiacal foreground is not different than for any other stars, thus the only term that is different is the stellar leakage.

Studies of the detectability of Earth like planets for TPF and Darwin indicate that the dominant noise term for the bulk of the detectable systems is the local zodiacal foreground emission and not the stellar leakage contribution. This is because the largest number of detectable planets is for stars that are the furthest away from the Sun. As a result, the contribution from stellar leakage is important only for the nearest stars. These conclusions are summarized in Fig. 5, which shows the projected Habitable Zone radius versus distance and displays the breakpoint in dominant noise contributions between stellar leakage and local zodiacal emission (see Dubovitsky \& Lay 2004).

Figure 6 displays the inner habitable zone radius versus distance for the subgiant and giant stars in our sample. It is clear from this figure that a similar breakpoint should exist, and therefore Earth like planets in the habitable zone could also be detected for these stars as well. It is also evident from the figure that a larger sample of such stars should be investigated because current designs for TPF have sufficient resolution and sensitivity.

\section{Conclusions}

We have shown that the evolution of the habitable zone as stars move off the main sequence and onto the subgiant and later the red giant branches of the HR diagram 
provide a new opportunity for the search for Earth-like planets and life. For these stars life has an opportunity to evolve under conditions with much less bombardment than existed in the early Solar system. We also show there are adequate numbers of such stars in the Solar neigborhood, and that preliminary analysis demonstrates that planets around such stars could be detected without any modifications to present architectural concepts for the interferometer version of TPF and Darwin.

\section{Acknowledgements}

We acknowledge support from GSFC Internal Research and Development and Director's Discretionary Funds, a Michelson Fellowship, a National Research Council (NRC) Fellowship, and support from the CNRS.

\section{References}

Arrhenius, S.A. 1903, in The Quest for Extraterrestrial Life, ed. D. Goldsmith (Mill Valley: University Science Books), 32

Beichman, C., Woolf, N.J., Lindensmith, C., ed. 1999 (JPL Publ. 99-3; Pasadena: Caltech)

Clampin, M., Ford, H.C., Illingworth, G., \& Petro, L. 2001 BAAS, 33, 1365

Danchi, W.C., Deming, D., Kuchner, M.J., \& Seager, S. 2003a ApJ, 597, L57

Danchi, W.C., et al. 2003b, (ESA Publ. SP-539; Noordwijk: ESA), 83

Forget, F. 1998 Earth, Moon, Planets 81, 59

Forget, F. \& Pierrehumbert, R.T. 1997 Science, 278, 1273

Fridlund, C.V.M. \& Gondoin, P. 2003 Proc. SPIE, 278, 1273

Gough, D.O. 1981 Sol. Phys., 74, 21

Dubovitsky, S. \& Lay, O.P. 2004 Proc. SPIE, 5491, 284

Kasting, J., Whitmire, D.P., \& Reynolds, R.T. 1993 Icarus, 101, 108

Lopez, B., Schneider, J., \& Danchi, W.C. 2005, ApJ, 627, 974

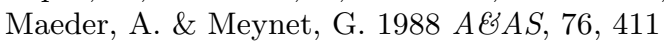

Mischna, M.A., et al. 2000 Icarus, 145, 546

O'Keefe, J.D. \& Ahrens, T.J. 1996 Science, 234, 346

Shu, F. 1982 The Physical Universe, (Mill Valley: University Science Books), 173

Trauger, J., et al. 2003 Proc. SPIE, 4854, 116

Weidemann, J. 1987 A $\& A$ A, 188, 74

Zubrin, R. 2001 J. British Interplanet. Soc. 54, 262

\section{Discussion}

DEN HARTOG: Giants are considerably larger in diameter than MS V stars, and in order to mitigate the extra leakage much shorter baselines are required: don't you run into trouble with collision avoidance requirements? On the other hand, the HZ are located further away from these stars: does the field of view limitation, coming in particular from the fiber coupling not restrict you too much in the assessment of the outer parts of the HZ?

DANCHI: Actually this is a common misconception because many astronomers are thinking of Asymptotic Giant Branch stars, which indeed are very large. However, since the subgiants and red giant stars are only modestly larger in diameter, our studies indicate this is not a problem except for perhaps a few of the closest ones. In answer to your second question, one could use a different fiber with a wider FOV for the closest such stars. For most of the sample it is not an issue. 